\title{
LES STRUCTURES D'UN GRAND COURS D'EAU A SALMONIDÉS: LA LOUE (MASSIF DU JURA) Essai typologique et problèmes des relations entre espèces et milieu'.
}

\author{
par J. VerneauX ${ }^{2}$ ol M. Rezzou, $K^{3}$.
}

Une étude typologique est effectuée sur la Loue, grand cours d'eau à Salmonidés du massif du Jura, à l'aide de la méthode d'analyse factorielle des correspondances.

Cette méthode est appliquée à l'analyse des variations longitudinales de 18 composantes physico-chimiques et de la distribution de 135 espèces de Poissons (18 espèces), Plécoptères (27 espèces), Êphéméroptères (33 espèces) et Trichoptères ( 57 espèces) sur 18 stations jalonnant les $126 \mathrm{~km}$ du cours d'eau.

Les structures biologiques sont déterminées séparément pour chaque ordre puis pour l'ensemble de la faune étudiée; 4 groupements et 12 noyaux d'affinités écologriques sont mis en évidence.

L'analyse mésologique fait apparaître une participation hiérarchiséc des paramètres mais aussi l'absence d'une structure abiotique nette soulignant le caractère intégrateur des espèces et faisant appel à la notion de «complexes paramétriques équivalents ».

Les résultats obtenus au niveau des espèces, du cours d'eau lui-même et des paramètres physico-chimiques sont commentés puis confrontés; l'intérêt de la recherche typologique est alors $m$ is en évidence parallèlement au caractère essentiellement relatif du déterminisme des espèces et de leurs groupements.

\section{Biological structure of a large salmonid river in the Jura mountain (the river Loue). \\ Typological data and interrelationships.}

Typological studies have been done on a large salmonid river in Jura mountain (the river Loue), by means of a factor analysis.

This method is used for the analysis of longitudinal variations of 18 physical and chemical properties and for studying the distribution of 135 freshwater species : Fish (18 species), Plecoptera (27 species), Ephemeroptera (33 species) and Trichoptera (57 species), located on 18 stations distributed all along the river $(126 \mathrm{~km})$.

1. Texte des communications présentées aux colloques de l'Association Française de Limnologie : Toulouse, 17 avril 1971 — Thonon, 25 mai 1972.

D'après l'un des chapitres d'une thèse de Doctorat d'Etat soutenue le 10 novembre 1973 à la Faculté des Sciences de Besançon.

2. Laboratoire d'Hydroécologie de la Division Qualité des Eaux, Pêche et Pisciculture du C.T.G.R.F.F., Paris et Laboratoire de Zoologie de la Faculté des Sciences de Besançon.

3. ex. $M^{110}$ Gaborit, Laboratoire de Statistique Mathématique de l'Université de Paris et Laboratoire de Statistique de l'Université de Rouen. 
The biological "structures" are determined separately for each order, then for all the fauna : 4 groups and 12 sub-groups of "ecological affinities"' are established.

The analysis of the abiotic environment indicates a hierarchical participation of the components but also the absence of a clear abiotic "structure" which conforms to the theory of the "equivalent ecological combinations" and emphasises the integral nature of the species.

The results obtained at the spccies level, from the water course, and from the physical and chemical components are discussed and compared. The value of the typological research is pointed out together with the relative character of the causation of the species and their groupings.

\section{NATURES DES RECHERCHES}

Avant d'entreprendre des recherches biotypologiques à l'échelle du bassin du Doubs, les méthodes et les techniques relatives à l'obtention, à l'expression et au traitement mathématique des données furent testées et adaptées au cours de travaux préliminaires effectués sur la Loue, le plus grand cours d'eau du bassin après le Doubs. Les principaux résultats obtenus, vérifiés de nombreuses fois par des campagnes de contrôle, font l'objet de la présente note.

Après qu'une étude synthétique préalable eut permis de définir les caractéristiques générales du cours d'eau et de choisir les stations, l'analyse méthodique du milieu (composantes) et des peuplements aquatiques (espèces) fut exprimée, à partir de deux fichiers, sous la forme de matrices de données (biologiques et abiotiques) dont il convenait de déterminer la méthode mathématique de traitement la mieux adaptée à la résolution du problème posé : la recherche d'organisations (structures) biologiques et mésologiques éventuelles et la mise en évidence de certaines relations entre espèces et milieu.

Bien qu'une analyse plus complète des peuplements, des diatomées aux poissons, soit en cours sur un certain nombre de stations du bassin, la durée d'un tel travail nous a conduit à limiter à 4 ordres (poissons et insectes benthiques) l'essai biotypologique préliminaire effectué sur la Loue par une recherche des corrélations entre les variations de 18 paramètres et la distribution de 135 espèces sur 18 stations jalonnant le cours d'eau (carte, figure 1). La mise en évidence d'une structure biologique typique est à l'origine de la proposition de certains éléments nouveaux relatifs à l'interprétation des résultats.

Après un exposé succinct des principales caractéristiques du cours d'eau et des paramètres étudiés, sont présentés successivement la démarche effectuée et les résultats obtenus dans le cas de la rivière Loue en tant que bases de l'établissement d'une typologie 
des cours d'eau du bassin, susceptible d'être progressivement étendue.

La phase causale est abordéc au cours d'un examen des relations possibles entre la structure biologique globale et un certain nombre de composantes de l'environnement abiotique.

\section{SITUATION ET CARACTERISTIQUES GÉNÉRALES DU COURS D'EAU}

\section{1. Éléments géomorphologiques.}

Longue de 126 kilomètres pour une pente moyenne d'environ $3 \%$, la Loue, dont le débit moyen annuel varic longitudinalement de 10 à $60 \mathrm{~m}^{3} / \mathrm{s}$, est un grand cours d'eau à Salmonidés des plateaux calcaires du Jura (600 - 200 mètres) dont l'Hydrologie est dominée par la présence d'un Karst important ; si son bassin versant topographique approché est d'environ $1900 \mathrm{~km}^{2}$, on peut estimer, selon Delarosière (1968), que son bassin hydrogéologique réel, tenant compte de la répartition des communications souterraines, est constitué de deux unités, l'une lithologique, groupant les hauts bassins du Doubs et de la Loue $\left(2217 \mathrm{~km}^{2}\right)$, l'autre structurale, se rapportant au bassin inférieur de la Loue $\left(1056 \mathrm{~km}^{2}\right)$.

Le cours d'eau naît à $595 \mathrm{~m}$ d'altitude d'une résurgence principale (débits extrêmes : 1 à $100 \mathrm{~m}^{3} / \mathrm{s}$ ) dans l'Astartien, alimentés par une série de pertes jalonnant les cours supérieurs du Doubs et du Drugeon ; d'autres émergences bordent la partie apicale de la vallée.

La Loue reçoit successivement la Brême alimentée par le plateau d'Ornans, le Lison également issu d'une résurgence, la Furieuse qui draine les marnes du Lias et le Keuper moyen, enfin la Cuisance, elle-même alimentée particllement par les pertes de la Furieuse (Fournier, 1919, 1923).

Les principales communications souterraines connues sont indiquées sur la carte de la figure 1 ; seul le cours inféricur, dans les alluvions de la plaine de Chaux, est exclu du domaine karstiçue.

Après une séric de chutes et de cascades, à l'emplacement d'un ancien cours souterrain entrecoupé de "gours», recouvert de tufs et jalonné de blocs d'effondrement, le cours d'eau encaisse profondément des méandres épigéniques dans le cañon des gorges de Nouailles, s'ouvre ensuite sur les plateaux et pénètre le flanc oriental du faisceau de Quingey dont il suit l'axe des plis avant d'en ressortir par le flanc occidental.

A partir de Quingey, les phénomènes de sédimentation prennent progressivement le pas sur ceux d'érosion; le cours d'eau déve- 


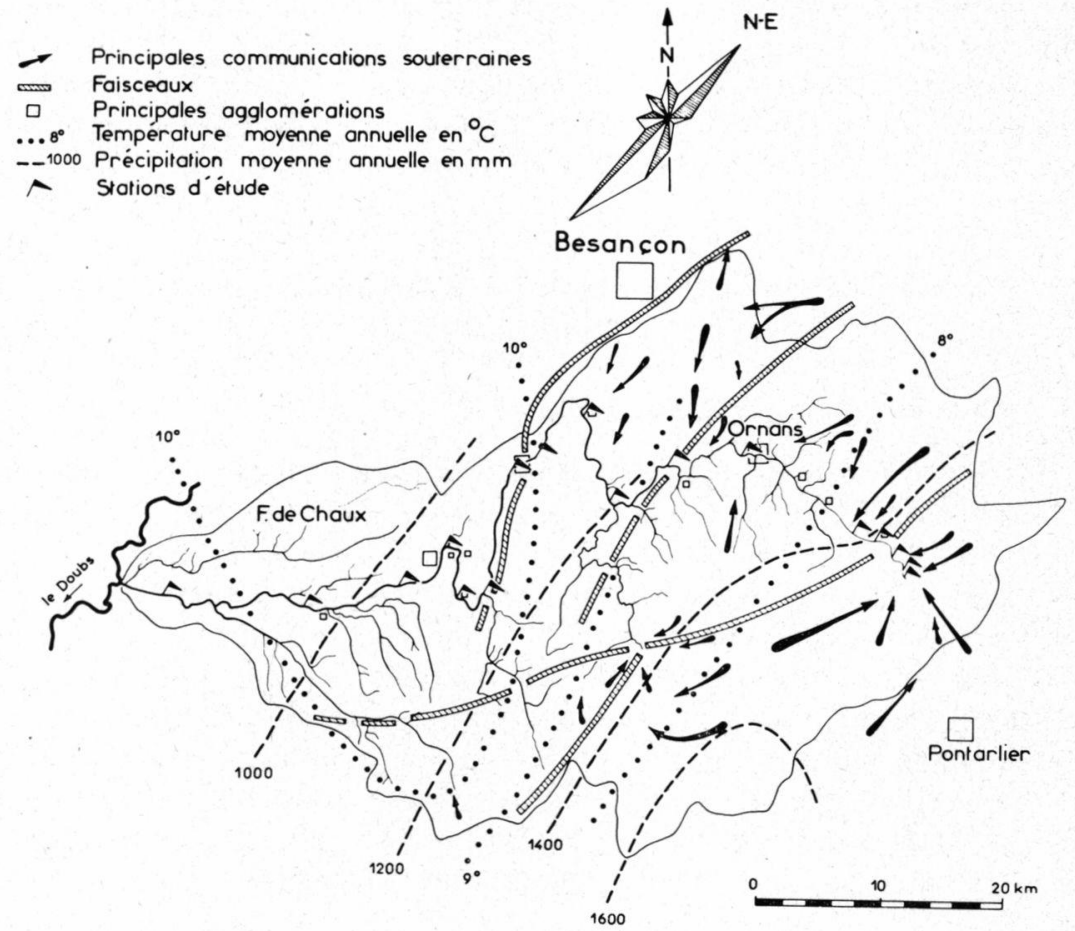

Fig. 1. - Le bassin de la Loue, caractéristiques générales, répartition des stations d'étude.

loppe alors ses méandres dans ses propres alluvions, reposant ellemême sur les dépôts siliceux témoins du passage d'un ancien «Aar-Doubs » pliocène (in Dreyfuss, 1960) et joint le Doubs, à 193 mètres d'altitude, en aval de Dole.

Toutefois, de récents travaux de redressement et de recalibrage de la basse vallée ont, de Chamblay au confluent, sensiblement modifié les caractéristiques morphologiques et dynamiques de ce secteur par suppression des habitats du faciès lénitique, augmentation de la pente et abaissement du niveau d'eau dans le lit luimême et dans la nappe (Dreyfuss, $1967 \mathrm{a}, \mathrm{b})$.

Les profils de la figure 2 expriment les variations longitudinales de la pente, du débit, de la température et de la section mouillée.

\subsection{Données climatiques et hydrologiques succinctes.}

Le climat du bassin présente un caractère continental souligné par une forte amplitude thermique annuelle et une insolation relativement intense ainsi qu'une tendance océanique marquée par une 


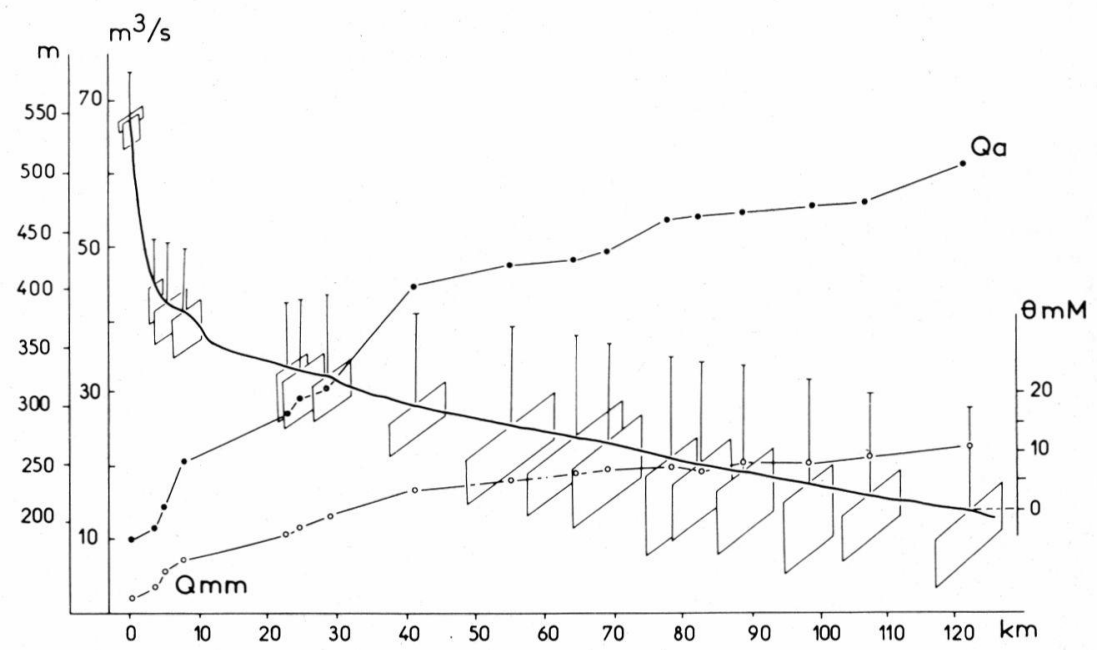

FIG. 2. - La Loue, profils topographique, hydrologique et thermique. Qa : débit moyen annuel ; Qmm : débit moyen minimum mensuel ; ${ }^{\theta} \mathrm{mM}$ : température moyenne maximum mensuelle.

pluviosité élevée et une répartition annuelle assez régulière des précipitations sous l'influence dominante des vents du sud-ouest.

En remontant le cours de la Loue, de la plaine de Chaux aux plateaux supérieurs, les moyennes annuelles passent de 900 à $1500 \mathrm{~mm}$ d'eau et de 10 à $7,5^{\circ} \mathrm{C}$ (courbes isohyètes et isothermes, moyennes 1961-1965, carte, figure 1).

La moyenne annuelle des précipitations (neige comprise), calculée sur 30 années est voisine de $1300 \mathrm{~mm}$; toutefois, on relève la présence d'un climat relativement sec et chaud au niveau de la basse vallée et celle, notée par Imchenetzky (1927-1928), d'un microclimat, de type rhodanien, particulier à la haute vallée abritée au creux d'un profond cañon.

Régime hydrologique (fig. 3).

La Loue, comme le Doubs et ses autres affluents, présente un régime mixte, pluvio-nival, dont le caractère jurassien est toutefois peu marqué étant donné l'altitude relativement faible du cours supérieur. L'influence nivale se traduit par un maximum de printemps et un étiage hivernal (rétention nivale) ; l'influence pluviale est marquée par un maximum en automne et un minimum en juillet( caractère océanique) ainsi que par une augmentation du débit en août-septembre (caractère continental peu marqué dans le cas de la Loue). 
Le graphique de la figure 3 représente les variations annuelles du débit moyen mensuel d'une année moyenne (1961-165) pour 3 stations jalonnant le cours d'eau : Mouthier (65), Lods (67) et Port-Lesney (76).

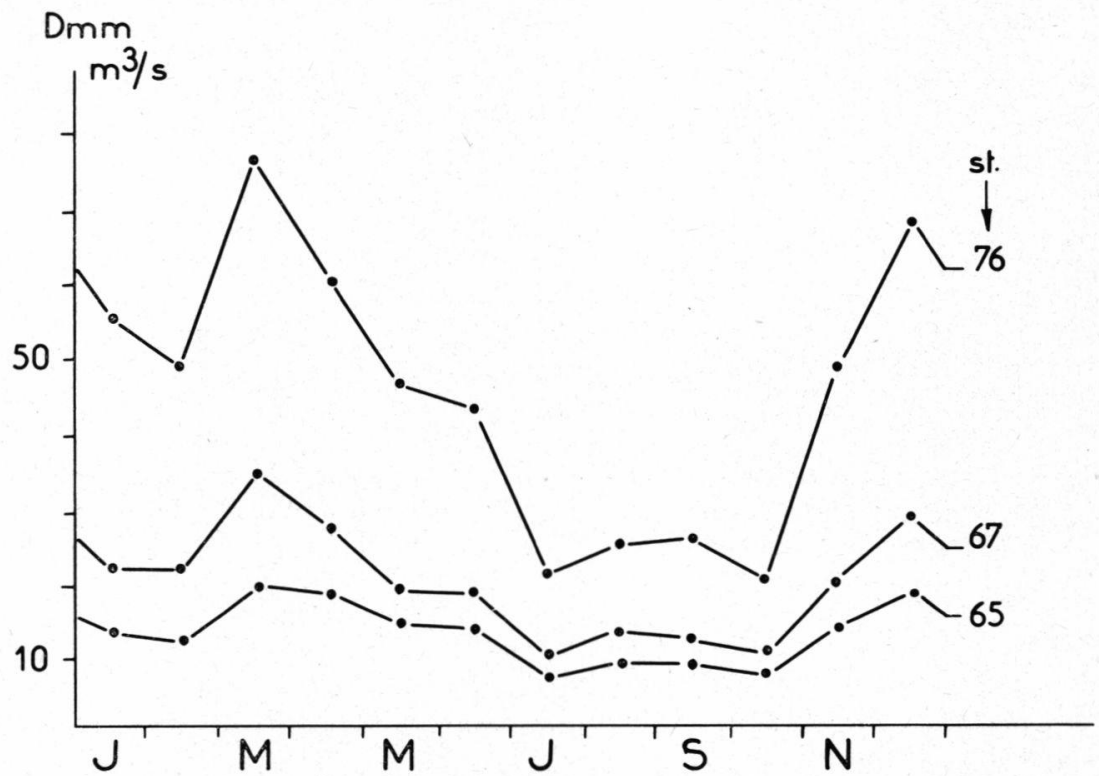

Fig. 3. - Régime hydrologique de la Loue, variations annuelles du débit moyen mensuel d'une année moyenne (1961-1965) sur 3 stations d'étude.

\subsection{Activité humaine et pollution.}

La Loue reçoit les eaux résiduaires d'une population d'environ 54000 habitants (densité : 29 habitants $/ \mathrm{km}^{2}$ ) à laquelle il convient d'ajouter la population des hautes vallées du Doubs et du Drugeon (environ 38000 habitants); actuellement les principales sources de pollution, en dehors des apports par lessivage des sols et de la pollution de la résurgence principale par les rejets de Pontarlier, sont constituées par l'agglomération d'Ornans, les confluences de la Furieuse (polluée par Salins-les-Bains) et de la Cuissance (polluée dès Mesney-Arbois) ainsi que par les agglomérations riveraines.

Une estimation chiffrée du « facteur pollution» selon la méthode des «indices biotiques» (Verneaux et Tuffery, 1967) permet de situer $56 \%$ du cours d'eau en classe $2,32 \%$ en classe 1 et $12 \%$ en classe 3 (les classes s'échelonnent de 1 à 5 dans l'ordre décroissant de la qualité biologique). 


\section{COMPOSANTES ETUDIEES.}

\section{OBTENTION ET EXPRESSION DES INFORMATIONS}

Effectuées de 1967 à 1972 , à raison de 3 , parfois 4 campagnes annuelles et au cours de prélèvements faunistiques mensuels ou hebdomadaires selon les saisons, les mesures el analyses ont porté sur 25 paramètres de l'environnement abiotique et 135 espèces de poissons et insectes à larves aquatiques. Les espèces de 4 ordres sont soumises à la présente analyse (poissons Téléostécns, Plécoptères, Éphéméroptères, Trichoptères - Verneaux, 1971 a - 1972) ; d'autres groupes sont en cours d'étude (Diplères Chironomides, Coléoptères, Cidonates, Mollusques, Crustacés, Vers) et les peuplements végétaux (macrophytes et algues benthiques) en cours d'analyse.

\subsection{Composantes physico-chimiques (mésologiques sensu stricto).}

Contrairement à une opinion assez largement répandue, la description du milieu s'avère généralement moins satisfante, plus discutable, que celle des peuplements ; nous n'en donnons qu'une certaine représentation, bien imparfaite, à la mesure des moyens techniques mis en euvre $^{1}$, conscients qu'un certain nombre de paramètres, peut-être essentiels, peuvent échapper à l'analyse.

Les cycles annuels n'ayant pu être établis pour tous les paramètres sur l'ensemble du bassin (123 stations) et dans le but de soumettre à l'analyse mathématique des données comparables, les informations portées sur la matrice initiale se rapportent à la même période du cycle annuel ; les valeurs numériques sont les moyennes diurnes relevées en période d'étiage ou de basses eaux au cours de six campagnes effectuées de 1967 à 1972 durant la période juillet-octobre. Ainsi, pour des raisons d'homogénéité des conditions d'analyse, sont retenus : le débit moyen du mois à plus faible débit et la température moyenne du mois le plus chaud (juillet pour ces deux paramètres) relevée dans le courant à $0,30 \mathrm{~m}$ de la surface de l'eau.

Des essais de traitement mathématique ont été effectués à partir des valeurs brutes puis à l'aide de classes ; cette dernière méthode permetlant d'obtenir des données plus homogènes a été finalement retenue. Cinq classes numériques sont utilisées pour repérer les variations de chaque paramètre à l'intérieur des limites constituées par les valeurs extrêmes, relevées dans les conditions définies précédemment.

1. Ces études ont été effectuées avec la collaboration technique de MM. Boucheron, Faessel, Lathelier, Rogger et Savary, techniciens supérieurs chargés des véhicules laboratoires et techniques du Ministère de l'Agriculture. 
18 paramètres physiques et chimiques, figurant sur le graphe de la figure 9, participent à l'analyse une fois exclus le B.V. Lopographique approché, peu significatif, le débit moyen annuel, ne correspondant pas aux conditions d'analyse et diverses variables associées utilisées pour le repérage des stations.

Le tableau suivant résume les principales caractéristiques physico-chimiques de ces eaux calciques, alcalines, fortement minéralisées, riches en oxygène dissous et très favorables au développement des organismes aquatiques (caractère eutrophe, sensu stricto).

Sont mentionnées les valeurs extrêmes relevées sur l'ensemble des stations dans les conditions précédemment définies, les variations ne suivant pas obligatoirement l'ordre des stations.

alt : $543-193 \mathrm{~m}-\mathrm{p}: 84-0,6 \%-\mathrm{Sm}: 5,7-65 \mathrm{~m}^{2}$

Qmm : $1,6-24 \mathrm{~m}^{3} / \mathrm{s}-\theta \mathrm{mM}: 9-18{ }^{\circ} \mathrm{C}-\mathrm{pH}: 7,2-8,2$

$\mathrm{C}: 310-386 \mu \mathrm{mhos} / \mathrm{cm} / \mathrm{cm}^{2}-\mathrm{Ca}-\mathrm{Mg}: 110-132 \mathrm{mg}$

$\mathrm{HCO}_{3}^{-}: 250-305 \mathrm{mg} / 1-\mathrm{Cl}^{-}: 7-8 \mathrm{mg} / \mathrm{l}-\mathrm{SO}_{4}{ }^{--}: 3-16 \mathrm{mg} / \mathrm{l}$ $\mathrm{PO}_{4}{ }^{--}: 0,01-0,60 \mathrm{mg} / \mathrm{l}-\mathrm{NO}_{3}^{-}: 0,55-3,21 \mathrm{mg} / \mathrm{l}$

$\mathrm{NO}_{2}^{-}: 0,00-0,09 \mathrm{mg} / \mathrm{l}-\mathrm{NH}_{4}{ }^{+}: 0,00-0,18 \mathrm{mg} / \mathrm{l}-\mathrm{O}_{2} 9,5-12,3 \mathrm{mg} / \mathrm{l}$

\% sat : $87-126$ - oxyd. $\left(\mathrm{KMnO}_{4}\right): 0,4-3,2 \mathrm{mg} / \mathrm{l} \mathrm{O}_{2}$

D.B.O. $: 5,7-4,0 \mathrm{mg} / 1 \mathrm{O}_{2}$.

\subsection{Les espèces'.}

Le répertoire des espèces, ayant fait l'objet de publications antérieures (Verneaux 1971 a, 1972), n'est pas reproduit ici ; toutefois, le lecteur peut aisément retrouver la nomenclature des espèces à l'aide des graphiques et de l'analyse des groupements. Participent à l'analyse : 17 espèces de Téléostéens, 27 Plécoptères, 34 Ephéméroptères et 55 espèces de Trichoptères.

L'obtention de données numériques représentatives relatives à la faune des cours d'eau constitue un problème examiné par la plupart des hydroécologistes (in Macan 1958, Tuffery 1971) sans que se soit dégagée une méthode d'échantillonnage quantitatif réellement préférentielle.

L'important est d'adapter la méthode utilisée à l'échelle de travail, qui est ici la station et non l'habitat, à la nature de la faune considérée ainsi qu'au traitement mathématique utilisé; dans le cas présent le critère présence-absence s'est révélé insuffisant et l'utilisation de données numériques brutes, inadaptée.

a) Les Poissons.

Diverses techniques d'inventaire furent utilisées (Verneaux, 1971 a) les captures au moyen de divers appareils de «pêche élec-

1. Les problèmes posés par la détermination des espèces ont pu être en partie résolus grâce à l'aimable collaboration de MM. Berthélemy, Décamps, Degrange, Sowa, Thomas et Vaillant à qui nous adressons nos vifs remerciements. 
trique »ayant été effectuées sur des aires de 10 à 50 ares prospectées selon la méthode de De Lury (1947).

Afin de permettre la comparaison de nombres d'individus, rapportés à une surface de 10 ares, appartenant à des espèces dont les capacités de reproduction et d'occupation de l'espace vital sont très différentes, nous avons utilisé 5 classes d'abondance relative ; chaque espèce possède sa propre abondance maximale représentée par le nombre d'individus capturés sur 10 ares de la station considérée comme optimale pour l'espèce dans le bassin étudié. Pour les petites espèces très abondantes (Vairon, Goujon, Loche franche, Ablette) les aires de pêche ont été très réduites et les dénombrements remplacés par des estimations.

b) Les inSECTES a LARVES AQUATIQUES.

Compte tenu de la grande variabilité des populations larvaires, locale (mosaïques de biotopes et de biocénoses), saisonnière (cycles de développement, phénologie) ou aléatoire (crues, dérive, etc.), la plupart des techniques de prélèvement furent mises à contribution (divers échantillonnages benthiques, élevages in situ et en laboratoire, pièges à imagos, chasses d'adultes...) dans le but d'obtenir une image la plus fidèle possible du peuplement de chaque station.

Les habitats des différentes stations furent prospectés durant 5 années à raison d'un inventaire benthique mensuel et d'une ou deux prospections hebdomadaires des nymphes et imagos entre avril et novembre jusqu’à ce que pratiquement toutes les espèces aient été reconnues dans leur abondance relative au niveau de chaque station. La troisième année, les espèces nouvelles pour le cours d'eau ne représentent qu'environ $15 \%$ du nombre total des espèces prélevées, les prospections suivantes permettant de préciser par élevage les déterminations délicates et de vérifier la validité des classes d'abondance retenues.

Comme pour les poissons, 5 classes d'abondance ont été déterminées; toutefois les valeurs numériques ne sont pas relatives à une surface ou à un volume donné mais sont obtenues en effectuant la somme des captures pouvant, avec certitude, être rapportées à la station considérée (larves et nymphes déterminables et adultes capturés in situ ou issus de la mise en élevage de substrats localisés).

La méthode retenue s'apparente à celles utilisées par d'autres écologistes d'eau courante (Berthélemy 1966, Décamps 1967, ...) bien que les données numériques relatives ne soient pas liées à

Nous nous garderions d'engager ici un débat sur la détermination de «l'espèce », notre travail en ce domaine s'étant limité à la reconnaissance, sous la même appellation, des formes morphologiques constantes et identiques ou reconnues comme telles. 
l'utilisation d'un seul mode de prélèvement mais du plus grand nombre possible et que les classes soient adaptées, par défaut, à chaque famille selon le procédé utilisé au niveau de chaque espèce pour les poissons.

Comme pour les composantes mésologiques, les résultats ont été groupés dans une matrice où les classes d'abondance relative figurent pour chaque espèce, au niveau de chaque station (Verneaux $1973)$.

\section{TRAITEMENT MATHEMATIQUE DES DONNÉES}

\subsection{Choix de la méthode d'analyse.}

Diverses méthodes d'analyse mathématique furent expérimentées parmi lesquelles, après l'essai de différents «tests d'affinité », plusieurs procédés de "classification automatique», permettant de définir des groupements d'espèces mais non les liens entre ceux-ci, puis «l'analyse en composantes principales »1, enfin «l'analyse des correspondances $\gg$ exposée avec précision et clarté dans les textes fondamentaux de Cordier (1969), Lebart (1969) et Benzecri (19701973).

Si les résultats obtenus par «l'analyse factorielle en composantes principales» (in Mœschler 1969) furent cohérents pour les espèces les plus fréquentes et permirent de dégager une structure biologique partielle, les groupements et les positions des autres espèces dans le système des 2 ou 3 premiers axes factoriels se révélèrent peu conformes aux réalités de terrain et parfois même aberrants (ainsi le Poisson-chat, la Truite arc-en-ciel, le Blageon et la Perchesoleil se trouvaient étroitement associés !).

En dehors du fait qu'une recherche typologique nécessite la prise en compte de toutes les espèces afin d'éviter le plus possible les choix arbitraires, la méthode précédente présente l'importante lacune, étant donné le but poursuivi, de ne pas établir les relations entre espèces et stations. "L'analyse des correspondances» finalement retenue permet de résoudre immédiatement ce dernier problème et les résultats obtenus peuvent être considérés comme bénéficiant d'un degré suffisant de validité en ce qui concerne l'agencement structural proprement dit (groupements espèces - stations et liaisons entre ces groupements) ; des incertitudes demeurent et des hypothèses sont formulées au niveau de l'interprétation des structures, au moment d'aborder la phase explicative, au cours de la confrontation des données biologiques et mésologiques (non au sens

1. Grâce à l'aimable concours de M. le Professeur Møeschler (C.R.A.R., Université de Genève) à qui nous adressons nos vifs remerciements. 
large proposé par Bertillon mais au sens restreint de composantes du milieu, sensu stricto).

Étant donné la complexité des relations de causalité dans les phénomènes biologiques, il est d'ailleurs logique de penser que l'absence de relations directes, simples et isolées, entre composantes et milieu constitue précisément un aspect fondamental de l'écologie.

Si l'intérêt de la méthode choisic dans la mise en évidence de structures, c'est-à-dire d'une organisation logique des rapports entre 《individus» et «caractères », n'est plus à démontrer, à la suite des travaux effectués les quatre dernières années, dans des branches aussi diverses que la Phytosociologie, la Taxonomie végétale (Brunerye, Gorenflot et Roux 1969, Blaise 1969, Gardou 1970, Gasquez et Bidault 1971), l'Hydrochimie (Cazes, Solety et Vuillaume 1970), la Géographie (Massonie, Mathieu et Wieber 1971), la Biologie marine (Binet, Gaborit et Roux 1972), la définition d'une «structure typique» n'a, à notre connaissance, pas encore été réalisée au cours d'études sur les systèmes d'eau courante.

Particularités de la méthode.

- Elle présente l'intéressante particularité d'extraire des données la distribution simultanée des sujets (stations) et des caractères (espèces) ; les axes factoriels (vecteurs propres de la matrice d'inertie) étant les mêmes dans les deux cas, on obtient une représentation unique pour les sujets et les caractères permettant de répondre en toute objectivité, à cette question essentielle : «qui a quels caractères »?

- Les proximités (distances) entre espèces et stations ou leurs groupements, apparaissent immédiatement sur un graphique à 2 ou 3 dimensions $\left(F_{1}, F_{2}, F_{13}\right)$ matérialisant les distributions obtenues et les proximités entre stations peuvent être interprétées en fonction des espèces proches ou associées.

- Deux espèces qui ont même profil mais des abondances différentes auront des projections confondues; cette particularité fort intéressante pour l'analyse commune de groupes différents de données (analyses globales) doit être en revanche compensée par l'examen de la matrice initiale au moment d'aborder la phase explicative.

\subsection{Analyse des résultats bruts, propositions interprétatives - Structure ichtyologique de la Loue.}

Les résultats du passage à l'ordinateur (Univac 1 108) se présentent sous la forme d'un tableau numérique donnant les coordonnées de chaque station et de chaque espèce par rapport aux axes 
déterminés par l'analyse factorielle ; les valeurs des «saturations» ont été établies pour les 5 premiers facteurs d'analyse numérotés dans l'ordre décroissant des pourcentages «d'inertie expliquée ».

Dans le cas présent le calcul dégage 2 axes nets selon lesquels « caractères» et «sujets»s'ordonnent avec un degré de sûreté appelé pourcentage d'inertie expliquée (extension de la notion de variance $)$ de $80,6 \%\left(\mathrm{~F}_{1}: 63,08 \%, \mathrm{~F}_{2}: 17,46 \%\right)$ soulignant l'existence d'une organisation logique nette dans les rapports espèces stations.

Les facteurs suivants présentent des pourcentages d'inertie faibles $(5,88-4,74$ et 3,03$)$.

L'exploitation de ces résultats s'effectue en 3 étapes successives :

a) Phase descriptive.

- La première démarche consiste à matérialiser l'espace abstrait par un graphique (fig. 4) dont les axes sont les deux premiers facteurs d'analyse (ou les 3 premiers lorsque cela est nécessaire).
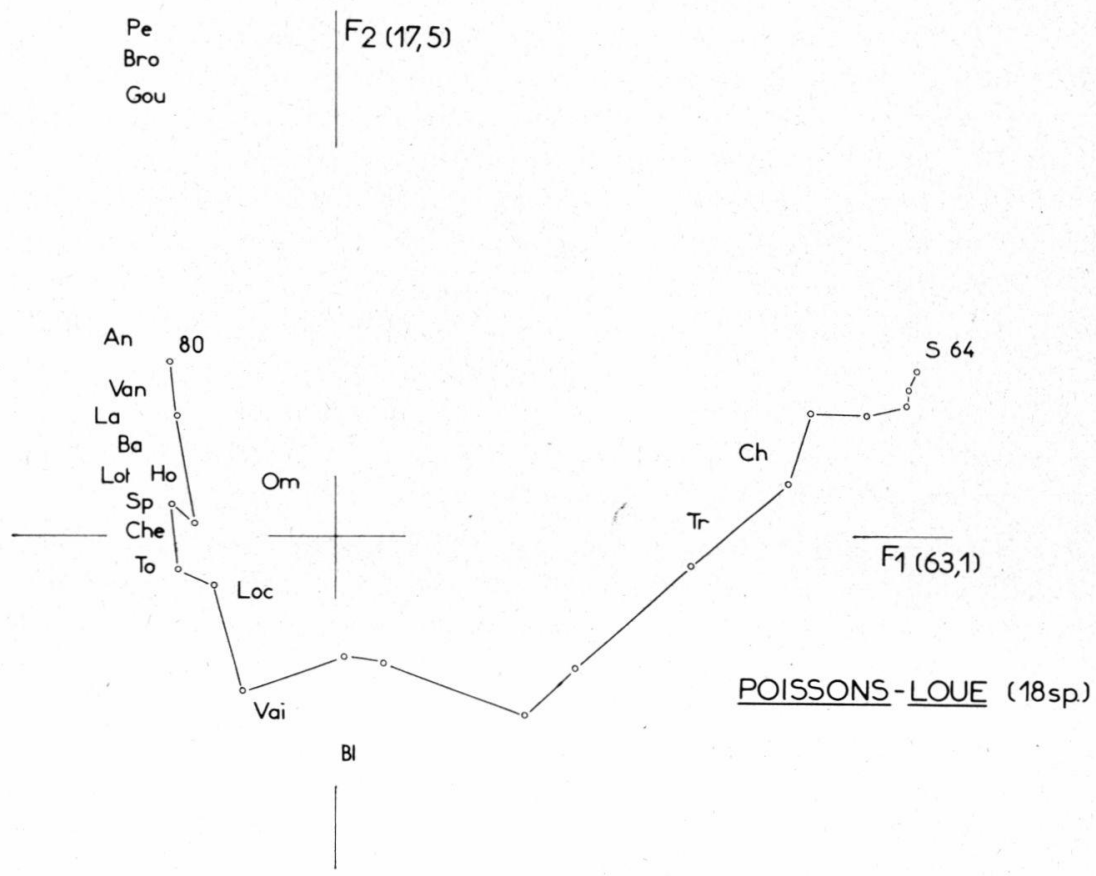

Fig. 4. - Analyse factorielle des correspondances espèces-stations : structure ichtyologique de la Loue dans le plan des deux premiers facteurs d'analyse. Les noms d'espèces figurent en abrégé sur le graphique : Ch :Chabot, Tr : Truite ... 

Ce double aspect de la signification des espèces est confirmé à la fois par les relevés de terrain et par le calcul des contributions relatives des espèces au plan $F_{1} / F_{2}$ qui permet de préciser la qualité de la représentation sur les axes d'analyse ainsi que la participation des espèces à l'établissement de la structure.

Ainsi contrairement au Chabot (Ch - 1), le Goujon (Gou - 14) apparaît comme une espèce très peu structurale bien que significative.

Au niveau de chaque groupement, les espèces seront nommées du centre à la périphérie et les espèces possédant les «participations structurales» les plus élevées seront portées en caractères gras.

Les espèces «typologiquement caractéristiques», selon les termes d'une étude de Lepneva commentée par Botosaneanu (1960), présentant les plus fortes contributions au plan $F_{1} / F_{2}$, sont à la fois proches de la courbe structurale et éloignées de l'origine des axes ; c'est le cas du Chabot $(\mathrm{Ch}-1)$, de la Truite $(\mathrm{Tr}-2)$, du Vairon (Vai - 3) et du Barbeau (Ba-11).

Inversement les espèces dont la contribution au plan $F_{1}-F_{2}$ es! faible, sont soit très largement réparties le long du cours d'eau et occupent des positions proches de l'origine des axes [espèces à fréquence élevée, eurytopes ou statistiquement peu discriminatoires dans le cas de la Loue, comme l'Ombre (Om-5), soit aléatoires ou accidentelles comme le Goujon (Go-14), le Brochet $(\mathrm{Br}-15$ ) et la Perche $(\mathrm{Pe}-16)$ et occupent des positions périphériques, éloignées de la courbe structurale.

Des calculs complémentaires ont montré que les contributions totales des espèces aux 5 premiers facteurs d'analyse sont très voisines des contributions au plan $\left(\mathrm{F}_{1}-\mathrm{F}_{2}\right)$ qui, présentant 80,6\% de l'inertie totale, exprime «l'essentiel»du phénomène par suite de l'existence d'une structure; de même si les contributions de certaines espèces aux deux premiers axes sont élevées (de 0,96 à $0,75)$ il n'y a pas d'espèces dont les contributions soient fortes sur les axes suivants $\left(0,32\right.$ est la contribution maximale sur $\left.F_{3}\right)$.

\section{Le problème des discontinuités.}

La distinction de noyaux d'espèces se succèdant dans un ordre donné le long de la structure, pose le problème fréquemment soulevé de l'existence de discontinuités ou de limites dans la distribution des espèces (Illies 1953, 1958, Berthélemy 1966, ...).

Les résultats obtenus, mettant en évidence l'existence de groupements distincts d'espèces parallèlement à une répartition des stations selon une courbe structurale, montrent clairement qu'il convient de distinguer l'établissement statistique de discontinuités entre les groupements d'espèces de l'expression résultante, sous une 
forme progressive continuc, de leur distribution le long du cours d'eau.

Nous distinguerons donc les accidents survenant dans la strueture biologique du cours d'eau, des limites statistiques permettant d'individualiser des «noyaux d'affinité structurale » d'après les distances séparant les espèces.

Trois accidents structuraux apparaissent sur ic graphique de la figure 4 : au niveau des s!ations 67-68, entre les stations 72 et 75 , puis au niveau des stations 75-78. Ces déformations structurales, retrouvées au cours de l'analyse des autres groupes faunistiques, expriment des perturbations (effets biologiques) dans la distribution des espèces dont les causes probables sont examinées après la confrontation des structures biologiques aux composantes de l'environnement abiotique.

\section{b) Phase explicative.}

La seconde phase de l'exploitation des résultats bruts, largement hypothétique, vise à découvrir la signification des axes qui nous est inconnue ; cette recherche explicative, partiellement effectuée par voie déductive à partir de l'édifice mathématique utilisé el de la confrontation des distributions des espèces (structures biologiques) et des paramètres, consiste surtout à formuler un certain nombre d'hypothèses afin d'éviter le piège des justifications empiriques.

Comme le souligne Massonie (1971), ces significations présumées, dépendant des rapports existants entre tous les caractères et tous les sujets, ne peuvent présenter qu'un caractère synthétique, essen. tiellement abstrait, exprimant un complexe de relations et non l'influence directe de l'un ou l'autre des éléments.

Une grande prudence est donc nécessaire au moment de formuler des hyptohèses suggérées par l'examen des facteurs supposés de la distribution des espèces (explication de la structure).

La recherche des correspondances entre espèces et paramètres est facilitée par la projection des paramètres sur les axes factoriels issus de l'analyse espèces- -stations.

Les propositions relatives à la signification des axes sont exposées après l'examen de l'analyse mésologique.

\section{c) Phase représentative.}

Une troisième démarche, non effectuée ici, consisterait à replacer les résultats obtenus (définis dans un espace abstrait) dans l'espace géographique initial représenté par le cours d'eau lui-même (Verneaux, 1973). 


\section{RESULTATS - STRUCTURES BIOLOGIQUES RELATIONS ENTRE ESPẼCES ET MILIEU}

\subsection{Au niveau des espèces : structures biologiques de la Loue.}

Les graphiques des figures 4 à 8 représentent, pour les 4 ordres analysés séparément (structures biologiques partielles) puis simultanément (structure biologique globale), les distributions structurales des espèces et des stations dans le plan des deux premiers axes.
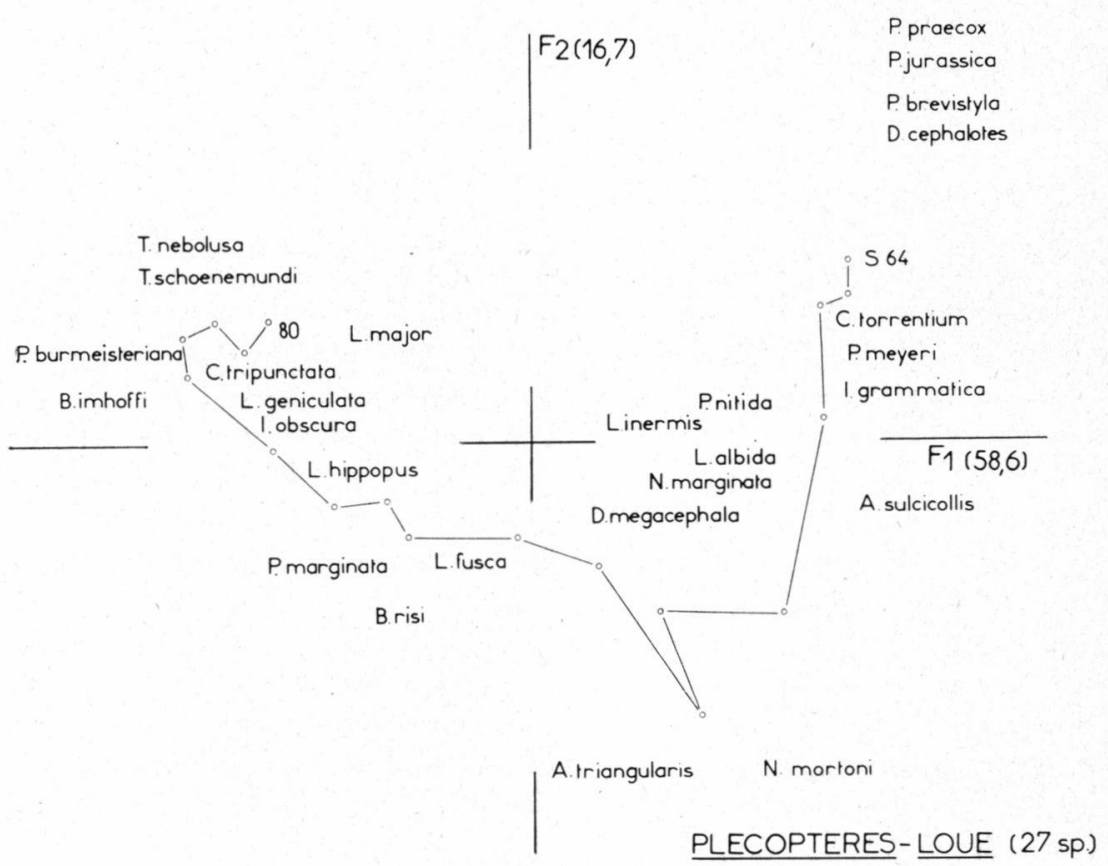

Fig. 5. - Analyse factorielle des correspondances espèces-stations : structure biologique de la Loue en fonction de la répartition des Plécoptères.

Six groupements s'individualisent le long de la structure en U, 6 espèces occupent des positions nettement périphériques (espèces à distribution aléatoire ou accidentelles) et 7 espèces (fréquentes, eurytopes pour la Loue) sont proches de l'origine des axes. On note l'existence des 3 accidents structuraux précédemment mentionnés (cf. fig. 4).

Quatre groupements s'individualisent assez bien malgré la présence de nombreuses espèces intermédiaires. Les espèces nettement 


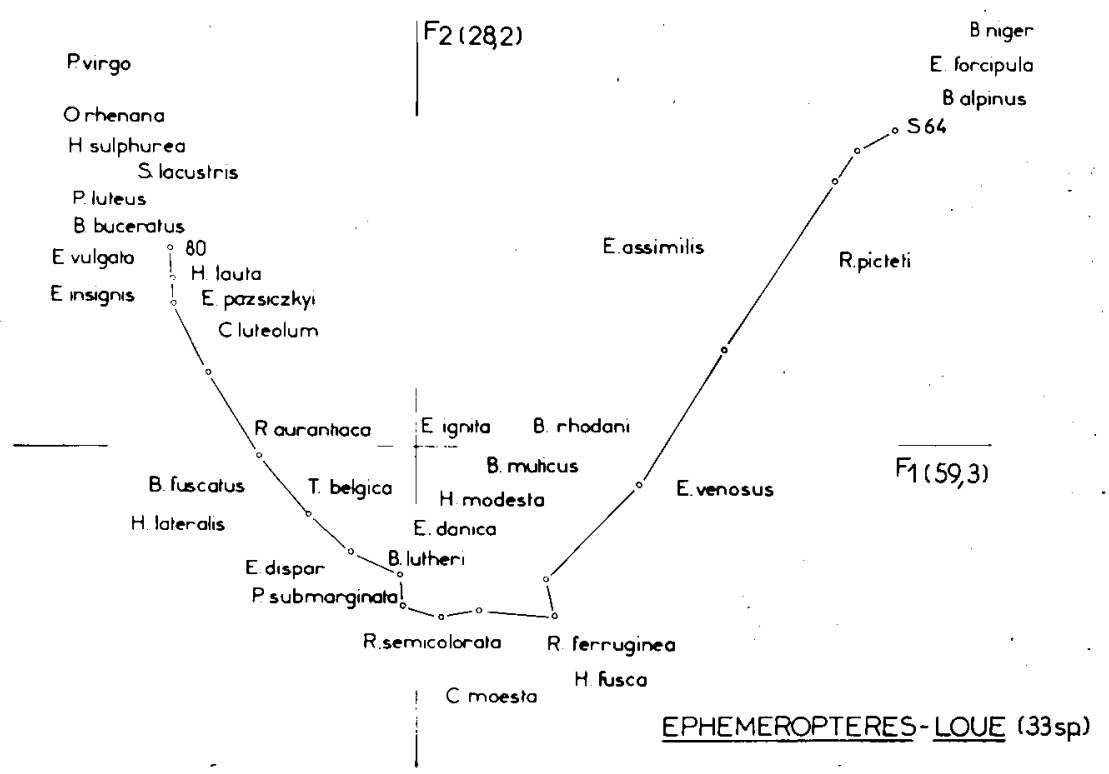

Fig. 6. - Analyse factorielle des correspondances espèces-stations : structure biologique de la Loue, en fonction de la répartition des Ephéméroptères.

périphériques sont rares mais les espèces centrales (eurytopes) abondantes (caractère général de l'ordre considéré).

Onze groupements se distinguent le long de la structure et le nombre des espèces repères, à forte participation structurale, est élevé (caractère général de l'ordre considéré).

Quatre groupements principaux et 12 noyaux d'affinités structurales se succèdent le long d'une structure biologique commune, progressive et continue à 2 accidents près ; les espèces de chaque noyau sont nommées du centre à la périphérie et les espèces structurales (espèces repères) figurent en caractères gras dans le répertoire suivant.

On distingue successivement :

- le groupement du secteur des résurgences $\left(G_{1}, 11\right.$ espèces $)$

- noyau 1-1 : Stenophylax mucronatus, Chaetopterygopsis maclachlani, Tinodes dives,

- noyau 1-2 : Rhyacophila pubescens, Perlodes jurassica, Protonemura praecox, Drusus mixtus, Crunoecia irrorata,

- noyau 1-3 : Dinocras cephalotes, Protonemura brevistyla, Plectronemia conspersa.

- le groupement du cañon des gorges de Nouailles $\left(G_{2}, 24\right.$ espèces $)$ 


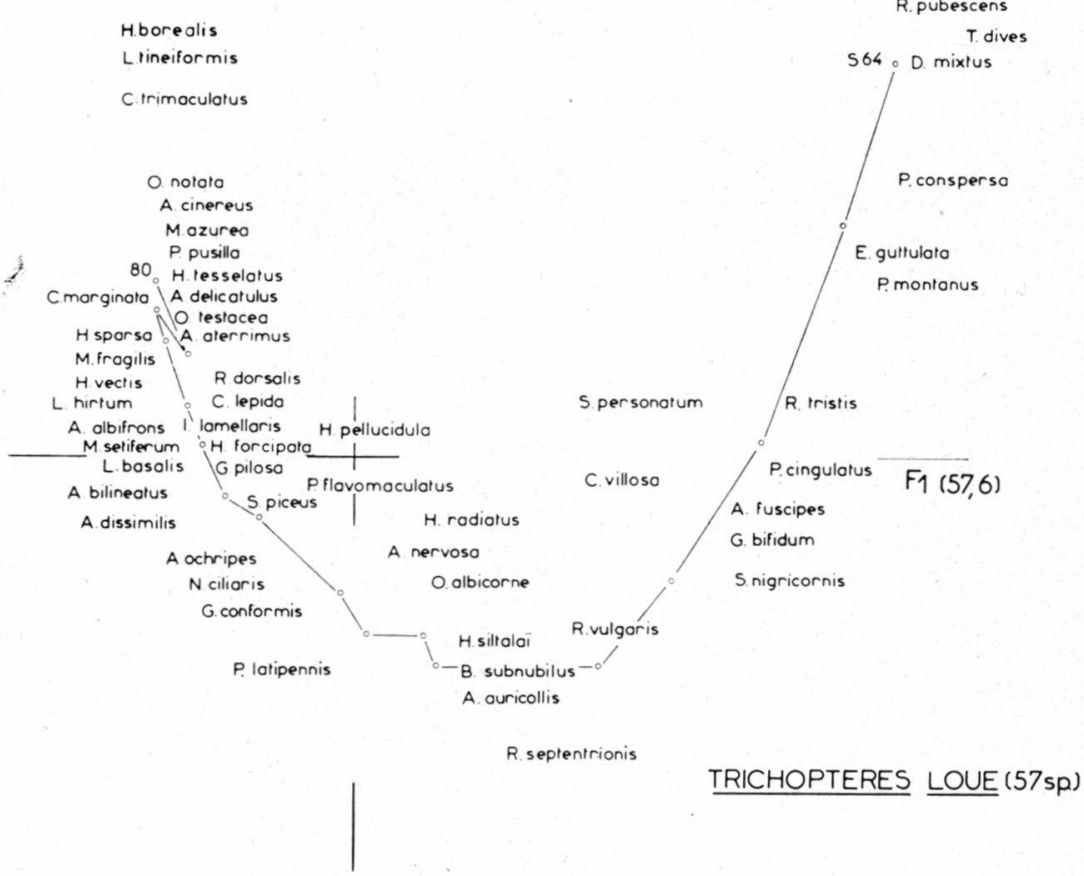

Fig. 7. - Analyse factorielle des correspondances espèces-stations : structure biologique de la Loue en fonction de la répartition des Trichoptères.

On distingue 3 noyaux et des espèces périphériques électives du secteur 2 mais s'étendant sur 1 et 3 : Protonemura nitida, Epeorus assimilis et Centroptilum luteolum,

- noyau 2-1 : Ecdyonurus forcipula, Ecclisopteryx guttulata, Philopotamus montanus, Baetis niger,

- noyau 2 - 2 : Isoperla grammatica, Baetis alpinus, Rhithrogena picteti picteti, Agapetus fuscipes, Protonemura meyeri, Potamophylax cingulatus, Rhyacophila tristis, Amphinemura sulcicollis. Glossosoma bifidum, Silo nigricornis, Chloroperla torrentium (en position intermédiaire entre $2-1$ et $2-2$ ),

- noyau 2-3 : Sericostoma personatum, Chaetopteryx villosa, Leuctra inermis, Leuctra albida, Nemoura marginata; ce noyau comprend Cottus gobio à sa partie inférieure. 


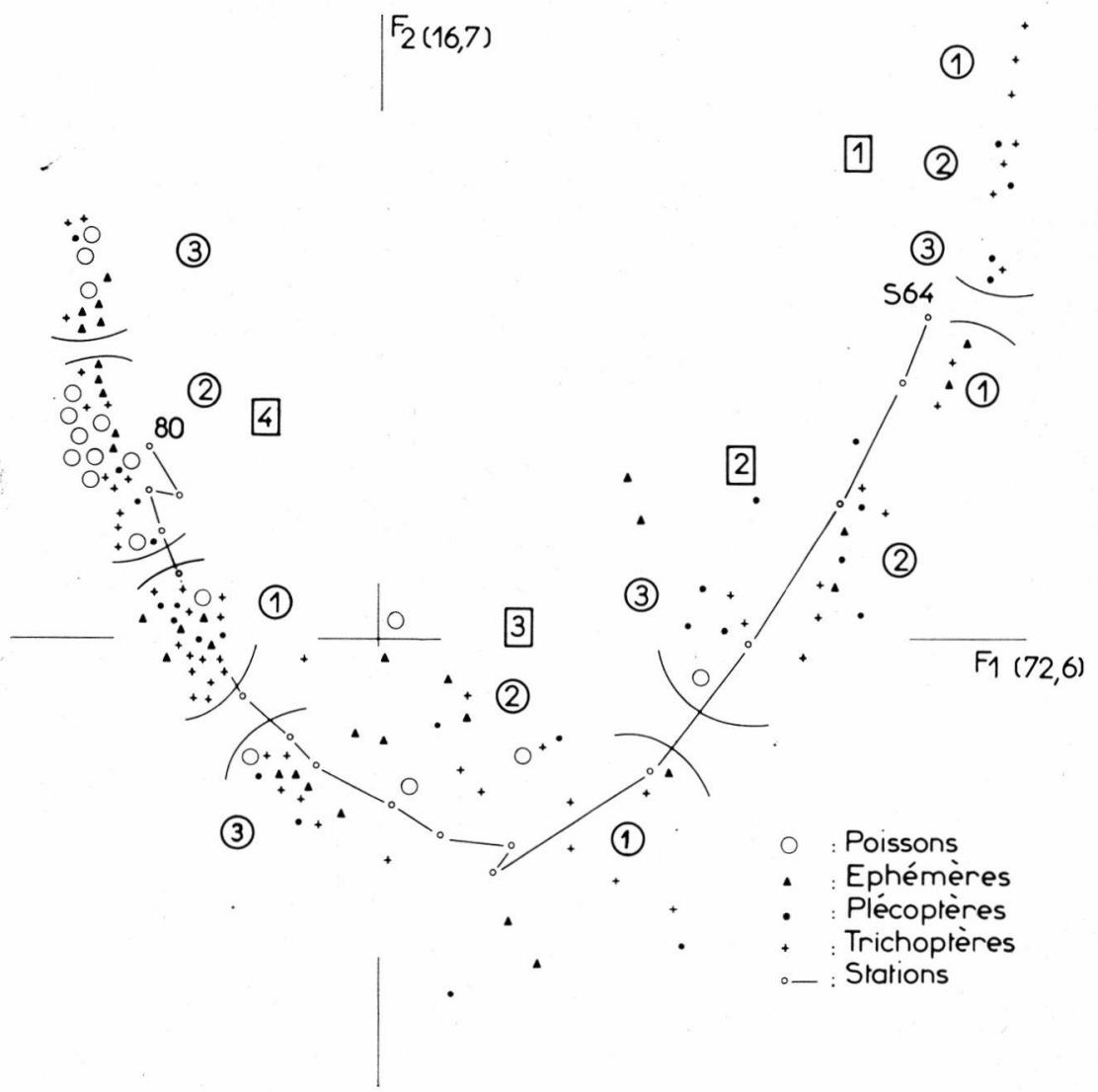

FIG. 8. - Analyse factorielle des correspondances espèces-stations : structure biologique globale de la Loue (plan $\mathrm{F}_{1} / \mathrm{F}_{2}, 89,3 \%$ d'inertie expliquée).

- le groupement du cours moyen $\left(\mathrm{G}_{3}, 38\right.$ espèces - Lods, Quingey) .

Ce groupement diffus, à l'exception du noyau 3 - 1, renferme une majorité d'espèces à faible signification structurale. La plupart de ces espèces, comme Ephemerella ignita, Odontocerum albicorne ou Dinocras megacephala, sont eurytopes à l'échelle du cours d'eau (largement distribuées des sources à l'embouchure) ; leurs affinités avec les autres espèces les classent au niveau d'un « écotone » ou secteur de transition entre deux zones écologiques caractérisées par des espèces à signification structurale plus forte.

D'autres espèces comme Amphinemura triangularis ou Habrophlebia fusca sont aléatoires et leur présence peut être liée à l'existence de biotopes particuliers (dépôts organiques); elles 
paraissent, par ailleurs, assez indifférentes vis-à-vis des autres espèces.

- noyau 3-1: on y distingue 4 espèces relativement structurales ; Ecdyonurus venosus, Rhyacophila vulgaris, Brachycentrus subnubilus, Allogamus auricollis et des espèces périphériques: Rhyacophila septentrionis, Nemoura mortoni, Rhithrogena ferruginea, Habrophlebia fusca, Amphinemura triangularis qui, sans être très fréquentes, ne manifestent pas, vis-à-vis des autres espèces, des affinités suffisamment fortes et constantes,

- noyau 3-2 : c'est un ensemble diffus d'espèces eurytopes (les plus fréquentes sur la Loue), la seule espèce relativement structurale étant le Vairon (Phoxinus phoxinus). Quinze espèces appartiennent à ce type ; Baetis muticus, Halesus radiatus, Leuctra fusca, Baetis rhodani, Hydropsyche siltalai, Odontocerum albicorne, Anabolia nervosa, Dinocras megacephala, Habroleptoides modesta, Ephemera danica, Ephemerella ignita, Hydropsyche pellucidula, Potamophylax latipennis.

La Truite (Salmo fario) et l'Ombre (Thymallus thymallus) appartiennent à ce groupement; cette dernière espèce, présente sur la Loue des gorges de Nouailles à la confluence avec le Doubs, occupe une position structurale assez proche de l'origine des axes. Sa présence, et plus encore son abondance, est liée à l'existence de faciès particuliers répartis tout le long du cours d'eau.

- noyau 3 - 3: ce groupement qui comprend le Blageon (Telestes soufia agassizi) constitue le seul noyau d'espèces affines pouvant être rapporté à la partie moyenne du cours d'eatu. Dis expèces sont groupées autour d'Ecdyonurus dispar : Baetis lutheri, Paraleptophlebia submarginata, Silo piceus, Notidobia ciliaris, Agapetus ochripes, Polycentropus flavomaculatus, Perla maxima, Brachyptera risi, Glossosoma conformis, Rhithrogena semicolorata.

- le groupement du cours inférieur $\left(G_{4}, 62\right.$ espèces) (lorsque les phénomènes de sédimentation prennent progressivement le pas sur ceux d'érosion à l'exception du secteur inférieur récememnt redressé).

- noyau 4-1: Leuctra geniculata Ecdyonurus pazsiczkyi, Torleya belgica, Heptagenia lateralis, Baetis fuscatus, Ithytrichia lamellaris, Isoperla rivulorum, Micrasema setiferum, Lasiocephala basalis, Athripsodes bilineatus, Hydroptila forcipata, Goera pilosa, Athripsodes dissimilis, Besdolus imhoffi, Isoperla obscura, Leuctra hippopus, Metalype fragilis, Cheumatopsyche lepida, Ryacophila dorsalis, Hydroptila vectis, Athripsodes albifrons, Hydroptila sparsa, Caenis moesta, Lepidostoma hirtum et la Loche franche (Nemacheilus barbatulus), 
- noyau 4-2: Ecdyonurus insignis, Habrophlebia lauta, Taeniopteryx schoenemundi, Mystacides azurea, Ephemera vulgata, Psychomyia pusilla, Perla burmeisteriana, Halesus tesselatus, Agapetus delicatulus, Oecetis testacea, Athripsodes aterrimus, Chimarra marginata, Oecetis notata, Leuctra major, Oligoneurilla rhenana; le Barbeau (Barbus barbus), l'Ablette spirlin (Spirlinus bipunctatus), le Hotu (Chondrostoma nasus), le Chevesne (Leuciscus cephalus), la Lotte (Lota lota), la Vandoise (Leuciscus leuciscus) et le Toxostome (Chondrostoma toxostoma) font partie de ce groupement,

- noyau 4-3 : ce noyau renferme une majorité d'espèces issues du Doubs : Siphlonurus lacustris, Polymitarcys virgo, Potamanthus luteus, Heptagenia sulphurea, Taeniopteryx nebulosa, Baetis buceratus, Cyrnus trimaculatus, Leptocerus tineiformis, Hydropsyche borealis ; le Goujon (Gobio gobio), la Perche (Perca fluviatilis) et le Brochet (Esox lucius) font partie de ce groupement d'espèces remontées du Doubs.

\subsection{Observations au niveau du cours d'eau.}

Les graphiques précédents montrent clairement que les limites statistiques, permettant d'individualiser des groupements successifs d'espèces, n'existent pas le long du cours d'eau, les seuils d'affinité ne correspondant pas, dans le cas de la Loue, à des discontinuités spatiales ; la structure typique est continue et progressive, les stations se succédant dans l'ordre de leur répartition des sources à l'embouchure. Toutefois, chaque cours d'eau possède sa physionomie propre et les accidents, se traduisant par des déformations de la ligne structurale, correspondent à des perturbations écologiques, artitıcielles dans le cas de la Loue.

Les accidents structuraux relevés sur les stations 68 et 78 correspondent respectivement à la pollution des eaux par l'agglomération d'Ornans et au redressement du cours inférieur de la Loue qui se traduit, au niveau des peuplements, par un "rajeunissement» artificiel du secteur allant de Chambley à la confiuence avec le Doubs. Ce sont les Plécoptères qui manifestent la plus plus grande sensibilité à ces variations des conditions mésologiques.

Une concavité de la courbe, particulièrement nette chez les Plécoptères, s'établissant entre les stations 69 et 74 est à rapporter à la fois à la médiocre qualité physico-chimique des eaux le long de ce secteur contaminé par une succession d'agglomérations secondaires (Scey-en-Varay, Chenecey-Buillon, Quingey, Port-Lesnay) et au passage du cours d'eau de la «montagne » à la plaine.

La fragmentation, mathématiquement illimitée, d'un cours d'eau en «zones» et «sous-zones»n'apparaît donc pas, contrairement 
à la détermination de groupements d'espèces électifs de biotopes clairement définis, comme une démarche préférentielle de la recherche typologique, dont l'objectif est de déterminer le «commun essentiel » biologique et mésologique au plus grand nombre possible de systèmes aquatiques; résultant de la confrontation des structures, la typologie sera d'autant plus généralisable que le nombre de structures confrontées sera grand.

\subsection{Analyse des correspondances entre paramètres et stations.}

Cette analyse dont les résultats sont exprimés dans le graphique de la figure 9 montre qu'il n'y a pas, pour $81,2 \%$ d'inertie expliquée, de répartition structurée (typique) des paramètres et des stations mais souligne l'existence d'une succession ordonnée approchée des stations le long du premier axe (projections sur $\mathrm{F}_{1}$ ).

Des groupements de stations, présentant un degré élevé d'affinité mésologique, apparaissent sur le graphique accompagnés des paramètres prenant à ces niveaux leurs valeurs les plus fortes. Ces

\section{$F_{2}(17,5)$}

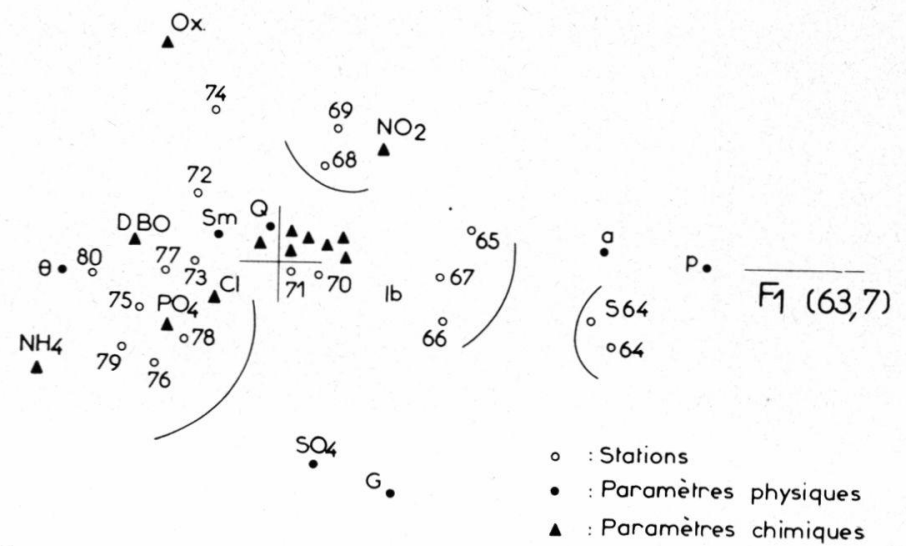

Fig. 9. - Analyse factorielle des correspondances paramètres-stations dans le cas de la Loue à partir des valeurs moyennes relevées en période d'étiage ou de basses eaux durant le mois le plus chaud (moyennes 1967-1972). 
composantes sont la pente et l'altitude au niveau des résurgences (S 64-64), l'indice biotique le long des gorges de Nouailles (stations 65-66-67), les nitrites au niveau d'Ornans (sta!ions 68-69), la section mouillée, le débit, la température, les chlorures, la D.B.O.. , les phosphates et les sels ammoniacaux au niveau du cours inférieur.

Cerlains paramètres chimiques, peu discriminatoires dans le cas de la Loue, par ailleurs fortement corrélés ou même redondants, se trouvent regroupés vers l'origine des axes ( $\mathrm{pH}$, conductivité, dureté, alcalinité, oxygène dissous) ; 3 paramètres occupent des positions périphériques (oxydabilité, sulfates et granulométric) et présentent un caractère local souligné par le fait qu'ils apparaissent au $3^{\prime \prime}$ et au $4^{\circ}$ facteur d'analyse $(9,2$ ei $4,3 \%$ d'inerlic expliquée) .

L'absence de structure esl soulignée par le fait que tous les paramètres participent également à l'explication des axes, les contributions étant le plus souvent très voisines; contrairement aux résultats obtenus pour les espèces, il n'y a pas de composantes à contribution nettement dominante, les plus fortes participations demcurant comprises entre 0,31 et 0,47 .

Toutefois ce calcul permet de proposer une hicrarchisation des composantes :

- sur le $1^{\text {er }}$ facteur d'analyse $\left(\mathrm{F}_{1}, 63,7 \%\right.$ d'inertie expliquée)

Les composantes dont les contributions sont les plus fortes sont dans l'ordre : la pente, la température el l'altitude,

- sur le 2 facteur d'analyse $\left(F_{2}, 17,5 \%\right.$ d'inertie expliquée), apparaissent le débit, les chlorures et les nitrates,

- sur le 3" facteur d'analyse $\left(F_{3}, 9,2 \%\right.$ d'inerlie expliquée) apparaissent successivement : la granulométric, les phosphates et les sulfates,

_ sur le 4" facteur d'analyse $\left(F_{4}, 4,3 \%\right.$ d'inertie expliquée), l'oxydabilité est préférentielle.

Cette porticipation hiérarchisée des paramètres à la distribution des stations étudiées est à rapprocher des remarques de Cummins et Lauff (1969) relatives au niveau d'intervention des composantes; les résultats précédents sont en accord avec la notion d'une intervention hiérarchisée des composantes (facteurs de «macro-» et de «micro-distributions» selon Cummins) qui s'effectuerait dans l'ordre des facteurs d'analyse, avec cette restriction qu'il n'est pas indiqué de considérer a priori l'un ou l'autre des paramètres examinés comme facteur déterminant l'absence, la présence ou l'abondance d'une espèce ou d'un groupement donné d'espèces. En fait cette hiérarchie se rapporte davantage à l'échelle dimensionnelle à laquelle s'exerce l'action qu'à l'action même des paramìtres sur les espèces. 
Par exemple, si l'on peut retenir que, dans le cas précis de la Loue, parmi les paramètres soumis à l'analyse et en tenant compte des valeurs de tous les paramètres, la pente, la température, l'altitude, le débit et les concentrations en chlorure et nitrates jouent un rôle prépondérant dans la distribution des stations et, selon le principe d'analogie, dans celle des espèces, il n'est pas possible de préciser le niveau d'action de chaque composante le long de la structure biologique et encore moins son rôle effectif au niveau des espèces ou de leurs groupements.

L'absence de structure abiotique, malgré une évolution longitudinale approximative selon le $1^{\text {er }}$ facteur d'analyse, indique que le déterminisme des biocénoses ne relève pas de relations simples entre tel ou tel paramètre et les espèces qui, comme l'a souligné Ulfstrand (1957), réagissent non pas à des facteurs mais à des "combinaisons de valeurs paramétriques». Il ne s'agit pas, bien entendu, de débattre de la validité du principe de causalité, ni du principe d'analogie qui en découle, mais de définir la nature des causes au niveau de l'espèce qui apparaît comme le complexe physiologique intégrateur qui précisément transforme des combinaisons de valeurs paramétriques en une structure biologique cohérente au niveau de l'écosystème.

\subsection{Discussion.}

a) Structures biologigues et significations.

La distribution des espèces et des stations dans les analyses partielles puis globale s'effectue dans l'ordre des stations, selon une courbe structurale en $\mathbf{U}$, dans le plan des deux premiers facteurs d'analyse qui représentent de 75,3 à $87,5 \%$ de l'inertie expliquée totale.

Cette structure biologique ne constitue ni une description de la répartition des espèces le long du cours d'eau, apparente à la lecture de la matrice des données, ni une méthode de détermination de biocénoses ou de «noyaux d'affinité cénotique, selon Bonnet (1964) où une même espèce peut figurer dans $\mathrm{N}$ groupements, mais une expression synthétique de l'ensemble des relations entre espèces et entre espèces et milieu.

La méthode utilisée qui, selon Bonnet (1966) «jette un pont entre l'étude des associations sans référence au milieu et celle des groupements établis en fonction du biotope»présente l'avantage de conserver le caractère global de l'environnement et de permettre le passage du stade descriptif à celui de l'interprétation (aspect causal). 
Chaque espèce ne figure qu'en un seul point (locus typologique) de la structure, déterminé par l'ensemble des relations entre espèces et stations; cette particularité souligne le caractère conceptuel et prévisionnel de la structure obtenue qui, dépassant le stade de la description se présente comme un «modèle». Vérifié, ce modèle est alors pourvu d'un caractère typologique net ... c'est-à-dire qu'il devient susceptible d'être généralisé et de permettre d'effectuer des « classements» aussi bien des espèces (positions typologiques) que des cours d'eau (structures).

\section{b) Propositions aU niveau des espèces.}

La méthode permet d'observer directement la succession structurale des espèces et de leurs groupements et d'évaluer l'intensité de leurs liaisons. La double appréciation des «distances» par rapport à l'origine des axes puis à la courbe permet de préciser le degré et la nature des «significations structurales» des espèces.

Deux notions ont été dégagées : la «contribution structurale » et le «degré de signification»; la première se rapport au système considéré, la seconde au concept typologique : ainsi Chloroperla torrentium (gpt. 2-1-2-2) est à la fois significative et structurale dans le cas de la Loue, Silo piceus (gpt. 3-3) est fortement structurale mais assez peu significative et Ephemerella ignita (espèce centrale) n'est ni structurale ni significative.

En d'autres termes, lorsque l'on se néfère aux espèces, l'examen de la position par rapport à la courbe structurale indique l'appartenance d'une espèce (ou d'un groupement d'espèces) à un « niveau typologique»donné et la position par rapport à l'origine des axes précise son degré d'euryécie relatif au complexe paramétrique considéré.

Ces deux notions permettent d'établir une hiérarchisation des espèces, de déterminer des «groupes écologiques» et d'aborder la définition des «spectres écologiques» en passant, à la suite de la confrontation des données biologiques et abiotiques, de la phase descriptive à la phase interprétative.

\section{Signification des groupements d'espèces.}

Chaque «niveau typologique » correspond à un groupement d'espèces typologiquement affines (biocénolypes), non obligatoirement cocénotiques, dont les caractéristiques écologiques sont voisines (relations, proximités ou distances vectorielles voisines vis-àvis des stations et de l'ensemble des autres espèces). Ces noyaux appartiennent aux «groupes écologiques statistiques tels qu'ils ont été définis par Duvigneaud (1946) et discuiés par Boudouresque 
(1971) qui remarque qu'un tel groupe «n'est jamais qu'une portion d'un continuum multidimensionnel ( nodum ») entre deux groupes écologiques». Ce modèle «a noda» (Poore 1962, Goodall 1969 ) permet d'interpréter l'organisation des peuplements de l'area et de passer du domaine du «perçu» à celui du «conçu» dans la mesure où la confrontation des analyses mésologiques et biologiques permet de préciser si l'eurytopie d'une espèce provient effectivement de son caractère euryèce (indifférence écologique) ou du fait que les milieux considérés (ici les stations) présentent des complexes paramétriques semblables ou équivalents.

\section{Relations avec la notion de "valence écologique ».}

On observe que, contrairement aux groupements intermédiaires se rapportant à un «écotone» les groupements extrêmes renferment une majorité d'espèces hautement significatives; ce fait, d'ailleurs prévisible, est à rapprocher des observations de Shadin (1935), Thienemann (1950), Bogoescu (1952), Ross (1956) puis Botosaneanu (1960) relevant des corrélations entre la valence, le spectre écologique et l'étendue de l'aire da distribution des espèces.

Il apparaît que pour un système aquatique donné, la Love en l'occurrence, les variations de la valence écologique en fonction de la distance aux sources, sont représentées par une courbe de Gauss approximative ; ceci, rappelé au moment d'examiner la signification des axes, conduit à effectuer des réserves vis-à-vis des relations pouvant être établies entre cette notion et divers degré de sténobiontie (ou d'eurybiontie) suivant les remarques formulées clairement par Sacchi et Testard (1971) qui après avoir distingué les notions de sénotopie et de sténoécie soulignent que « la correspondance entre l'étendue de l'aire de distribution et la valence écologique d'une espèce n'est ni absolue ni constante».

Dans le domaine des eaux courantes, en particulier chez les auteurs précédemment mentionnés, certaines conclusions ne se rapportent qu'à la branche ascendante de la courbe de Gauss du fait de la brièveté et du caractère montagnard des cours d'eau étudiés; seule la généralisation d'observations locales se trouve mise en cause.

\section{c) Aptitude typologigue au niveau des ordres.}

La confrontation des structures biologiques obtenues, précisée par l'examen des contributions des espèces au plan $\mathbf{F}_{1} / \mathbf{F}_{2}$ dans l'analyse globale, met en évidence, comme pour les espèces, une hićrarchisation des ordres étudiés selon leur plus ou moins grande aptitude typologique (nombre d'espèces structuralcs et significatives). 
Les Trichoptères viennent en tête suivis des Plécoptères pour les secteurs supérieurs, des Éphéméroptères pour les secteurs inférieurs, puis les Téléostéens ; la confrontation d'un plus grand nombre de groupes (des diatomées aux poissons) est nécessaire pour vérifier l'hypothèse d'une relation directe de cette aptitude avec la dépendance spatiale des espèces. La faculté des espèces d'effectuer des choix, c'est-à-dire d'intégrer des situations écologiques diverses, apparaît comme une manifestation des phénomćnes d'adaptation qui joueraient un rôle important dans la distribution des espèces à l'échelle du cours d'eau ou même de la station, sinon à celle de l'habitat où les phénomènes de compétition et de prédation interviendraient de façon prépondérante chez les invertébrés dans le déterminisme des biocénoses (Wautier 1949).

Nous retrouvons là encore le rôle important de l'échelle dimensionnelle souligné par Dussart (1966).

\section{CONCLUSIONS ET HYPOTHẼSES EXPLICATIVES}

L'analyse factorielle des correspondances a permis de mettre on évidence l'existence d'une structure biologique typique, susceptible de généralisation, donc une espérance biotypologique effective; les groupements typologiques peuvent être individualisés et repérés à l'aide des espèces dont la signification écologique globale est appréciée.

La phase suivante des études a consisté à vérifier l'existence d'une telle structure pour des cours d'eau très différents puis à l'échelle d'un réseau hydrographique complet (12 cours d'eau du bassin du Doubs) considéré comme unité géographique et fonctionnelle ; cette extension de la structure aboutit au concept typologique proprement dit, prévisionnel et déjà fonctionnel. Les mécanismes, la physiologie d'un tel système, peuvent alors être étudiés relativement à des niveaux typologiques de référence (Verneaux 1973 ).

Cette organisation biologique structurée ne correspond pas à une structure mésologique de même type mais les correspondances relevées permettent d'examiner les significations probables des deux premiers facteurs d'analyse :

- sur le $1^{\text {er }}$ axe, on observe une distribution ordonnée des stations des sources à l'embouchure tant au cours des diverses analyses biologiques qu'au cours de l'analyse mésologique; cette distribution, qui exprime le phénomène fondamental du remplacement longitudinal progressif des espèces les unes par les autres considéré comme l'une des plus anciennes acquisitions de la Lim- 
nologie (Décamps 1968), prend ici une existence conceptuelle et non plus seulement descriptive et correspond aux variations longitudinales progressives des valeurs paramétriques.

Ce premier facteur, considéré comme exprimant la "complexité croissante " du milieu par Massonie (1971) nous parait davantage traduire l'évolution progressive du milieu et corrélativement des peuplements.

- sur le $2^{\mathrm{e}}$ axe s'effectue un aller et retour particulièrement nct et constant dans les structures biologiques ; il pourrait correspondre, comme l'indique Massonie aux variations de la notion synthétique de «stabilité».

Plutôt que cette interprétation dynamique, historique, nous préférons retenir pour l'instant, la notion descriptive, spatiale, d'hétérogénéité du milieu correspondant à la complexité des peuplements, maximum au niveau du secteur médian (écotone).

Ces propositions, en accord avec le premier principe de Thiensmann (1920), de Gause ou de Monnard (1920), sont soulignées par la confrontation des matrices de données et par le fait que les s'ations appartenant au secteur intermédiaire, riche en espèces eurytopes (stations 68 à 79 de la structure biologique globale) occupent des positions centrales dans l'analyse mésologique; il est intéressant de noter également la cohérence de cette hypothèse avec les remarques précédemment effectuées au sujet de la valence écologique des espèces.

Aux complexes mésologiques où chaque facteur agit sur tous les autres et est, à l'inverse, affecté par chacun d'eux » (Wautier 1949) correspond, pour un cours d'eau donné, une structure biologique typique, aux accidents près, pouvant être définie par deux facteurs synthétiques principaux exprimant l'évolution progressive $\left(F_{1}\right.$ dominant) et l'hétérogénéité du milieu $\left(\mathrm{F}_{2}\right)$.

Ces résultats sont en accord avec la conception relativiste précédemment mentionnée dans laquelle, au niveau de l'espèce, la notion de «facteur déterminant» tend à être remplacée par celle de «combinaisons paramétriques équivalentes"; ces possibilités d'existence, sinon de cohabitation, se situent non à l'échelle des habitats mais des "niveaux écologiques », non à l'échelle des biocénoses mais à celle d'entités biologiques spécifiques plus larges et possédant une signification particulière liée au concept typologique appelées «biocénotypes» (Verneaux 1971 b, 1973)

La structure proposéc constitue une réponse affirmative à la question posée par Berthélemy (1966) qui, constatant le phénomène du remplacement des espèces pour plusieurs groupements d'espèces voisines, se demandait «s'il était possible de concevoir un schéma général dans lequel la position de chaque espèce serait définie par rapport à celles de toutes les autres». 
A la suite des nombreux auteurs qui, de Lepneva (1927) à Ikonomov (1970) ou Ko'wnacka (1972) ont tenté d'ordonner certaines « régularités» relevées parmi la diversité et la variabilité des systèmes d'eau courante et après les schémas proposés par Hesse (1924), Huet (1949-1962), puis Illies et Botosaneanu (1963) nous avons tenté de définir une démarche typologique consistant à rechercher une organisation susceptible d'intégrer dans l'espace abstrait les diverses répartitions, zonales ou non, régulières ou irrégulières, pouvant être relevées le long des systèmes d'eau courante d'une zone biogéographique donnée.

Cet «essentiel» commun à des milieux étudiés globalement apparaît, au terme d'une analyse qui est en réalité synthèse, sous la forme d'un modèle qui selon Benzecri (1969) «suit les données et non l'inverse $»$ dont il convenait de vérifier la validité à l'échelle d'un réseau complexe (le bassin du Doubs, Verneaux 1973) avant d'envisager son application à des zones biogéographiques plus étendues.

\section{TRAVAUX CITES}

Benzecri (J.-P.). 1969. - Introduction aux problèmes de typologie. Publ. Lab. Stat. Math. I.S.U.P., Paris, 5 p.

BenzeCRI (J.-P.). 1970. - Distance distributionnelle et métrique du «chi deux» en analyse factorielle des correspondances. $3^{\circ}$ éd., cours Lab. Stat. Math. I.S.U.P., Paris, 86 p.

Benzecri (J.-P.) et coll. 1973. - L'analyse des données, 1 : la Taxinomie. Dunod éd., 615 p.

Berthélemy (C.). 1966. - Recherches écologiques et biogéographiques sur les Plécoptères et Coléoptères d'eau courante (Hydraena et Elminthidae) des Pyrénées. Annls Limnol., 2 (2) : 227-458.

Binet (D.), Gaborit (M.) et Roux (M.). 1972. — Copépodes pélagiques du plateau ivoirien - I : utilisation de l'analyse des correspondances dans l'étude des variations saisonnières. Cah. O.R.S.T.O.M. Océanogr. (sous presse).

BLAISE (S.). 1969. - Considérations biosystématiques sur le groupe Myosotis discolor. C. R. Acad. Sc. Paris, 268 (D) : 2682-2685

Bogoescu (C.). 1952. - L'influence de la température et de la vitesse de l'eau sur la répartition des espèces d'Éphéméroptères. Rev. Univ. C. I. Parhon. Ser. Stiintelor Naturii, 1 : 172-177.

Bonnet (L.). 1964, - I.e peuplement thécamœehien des sols. Rev. Ecol. Biol. Sol., 1 (2) : 123-408.

BonNeT (L.). 1966. - Un aperçu de l'utilisation des méthodes statistiques en Biocénotique et en Ecologie : le problème des affinités. Ann. Stat. Biol. Besse-en-Chandesse, $1: 29-40$.

Botosaneanu (L.). 1960. - Sur quelques régularités observées dans le domaine de l"fecologie des Insectes aquatiques. Arch. hydrobiol., 56 (4) : $370-377$.

Boudoureseve (C.-F.). 1971. - Méthodes d'étude quantitative du Benthos (en particulier du Phytobenthos). Tethys, 3 (1) : 79-104. 
Brunerye (L.), Gorenfrot (R.) et Roux (M.). 1969. - Application de l'analyse factorielle des correspondances au traitement numérique de populations de Senecio helenitis (L.) Cuf. Rev. Géné. Bota., $76: 217-256$.

Cazes (P.), Solety (P.) et Vuillaume (Y.). 1970. - Exemple de traitement statistique de données hydrochimiques. Bull. B.R.G.M., 4 (3) : $75-90$.

Cordier-Escofier (B.). 1969. - L'analyse factorielle des correspondances. Publ. Scient. Litt. Louis-Jean, Gap, 59 p.

Cummins (K. W.) et Lauff (G. H.). 1969. - The influence of substrate particle size on the distribution of stream macrobenthos. Hydrobiologia, 34 (2) : 145-181.

Décamps (H.). 1967. - Écologie des Trichoptères de la vallée d'Aure (Hautes-Pyrénées). Annls Limnol., 3 (3) : 399-577.

Décamps (H.). 1968. - Vicariances écologiques chez les Trichoptères des Pyrénées. Annls Limnol., 4 (1) : 1-50.

Delarozière (0.). 1968. - Contribution à l'étude du bassin du Doubs - Climatologie, Hydrologie et déficit d'écoulement rapportés aux unités géologiques. Thèse 3" cycle, Besançon, S.G.R. Jura-Alpes, Lyon, 100 p. + ann.

DE LuRY (D. B.). 1947. - On the estimation of biological populations. Biometrics, 3 (4) : 145-167.

Dussart (B.). 1966. - Limnologie. Gauthiers-Villars éd., Paris, 676 p.

Duvigneaud (P.). 1946. - La variabilité des associations végétales. Bull. Soc. Roy. Bot. Belg., 58 : 107-134.

Dreyfuss (M.). 1960. - Caractères généraux et histoire géologique du Jura. Bull. Union Natur. Enseign. Public, 2 : 4-15.

Dreyfuss (M.). 1967 a. - Observations faites en 1966 vers le confluent de la Loue et du Doubs. Bull. Soc. Hist. Nat. Doubs, 69 (1) : 19-20.

Dreyfuss (M.). 1967 b. - Rivières droites et à méandres : essai d'identification de leurs caractères de stabilité. Ann. Scient. Univ. Besançon, Géol., 3 : 43-50.

Fournier (E.). 1919. - Gouffres, grottes, cours d'eau souterrains, résurgences, exsurgences et sources du département du Doubs. Besancon, 303 p.

Fournien (E.). 1923. - Grottes et rivières souterraines. Besançon, 180 p.

GaRdou (C.). 1970. Recherches biosystématiques sur la section jacea Cass. et quelques sections voisines du genre Centaurea L. en France et dans les contrées limitrophes. Thèse, Paris, $258 \mathrm{p}$.

Gasquez (J.) et Bidault (M.). 1971. - Sur l'utilisation simultanée des caractères qualitatifs et quantitatifs dans l'analyse factorielle des correspondances en Taxonomie végétale. C. R. Acad. Sc. Paris, 272 (D). 3024-3027.

Goodald (D. W.). 1969. - The nature of the mixed community. Proc. ecol. soc. Austr., $1: 84-96$.

Hesse (R.). 1924. - Tiergeographie. G. Fischer éd., Jena, 613 p.

Huet (M.). 1949. - Aperçu des relations entre la pente et les populations piscicoles des eaux courantes. Rev. Suisse Hydrol., 11 (3-4) : 322-351.

Huet (M.). 1962. - Influence du courant sur la distribution des poissons dans les eaux courantes. Rev. Suisse Hydrol., 24 (2) : 412-432.

Iкомомоv (P.). 1970. - Répartition des larves d'Éphéméroptères et Plécoptères dans les cours d'eau de Macédoine. Instit. Biol. Zool. Fac. Sci. Univ. Skopié, 22 : 1-11. 
ILLIES (J.). 1953. - Die Besiedlung der Fulda (insbes das benthos der Salmonidenregion) nach dem jetzigen Stande der Untersuchung. Ber Limnol. Fluss. Freudenthal, 5 : 1-28.

ILJIES (J.). 1958. - Die Barbenregion mitteleuropäischer Fliessgewässer. Verh. int. Ver. theor. angew. Limnol., 13 : 834-884.

Ilidies (J.) et Botosineanu (L.). - Problèmes et méthodes de la classification et de la zonation écologique des eaux courantes considérées surtout dlu point de vue faunistique. Mitt. int. Ver. ther angew. Limnol., $12: 1-57$.

ImChenetzky (M.). 1927-1928. — Les associations végétales de la partie supérieure de la vallée de la Loue. Bull. Soc. Hisl. Nat. Doubs, $35: 1-60 ; 36: 61-112$.

KowNACKA (M.) et KowNACKI (A.). 1972. - Vertical distribution of zoocenoses in the streams of the Tatra, Caucasus and Balkans Mts. Verh. Internat., Verein. Limnol., 18 : 742-750.

LeBart (L.). 1969. — Introduction à l'analyse des données : analyse des correspondances, validités des résultats. Consom. Ann. C.R.E.D.O.C., $4: 65-87$.

Lepneva (S. G.). 1928. - Les larves des Trichoptères de la région d'Olonetz. Trav. Expéd. Sci. Olonetz, 6 (Zool, 5) : 1-125.

Macan (T. T.). 1958. - Methods of sampling the bottom fauna in stony streams. Mitt. int. Ver. theor, angew, Limnol., $8: 21 \mathrm{p}$.

Massonie (J.-Ph.), Mathieu (D.) et Wieber (J.-C.). 1971. - Application de l'analyse factorielle à l'étude des paysages. Cah. Géograph. Besançon, $4: 50$ p.

MaschisR (P.) et SCheld.horn (J.-P.). - Recherche fondamentale et analyse régionale - Projet d'enquête dans le «Clos-du-Doubs» (Jura bernois, Suisse). C.R.A.R. Inst. Anthropol. Univ. Genève, $140 \mathrm{p}$.

Monard (A.). 1920. - La faune profonde du lac de Neuchâtel. Bull. Soc. Neuchâtel Sci. Nat., 44 : 65-236.

Poone (M. E. D.). 1962. - The method of successive approximation in descriptive ecology. Adv. ecol. res., 1 : 35-58.

Ross (H. H.). 1956. - Evolution and classification of the mountain Caddisflies. Univ. Illinois Press, Urbana, 213 p.

Sacchi (C. F.) et Testard (P.). 1971. - Ecologie Animale - organismes et milieu. Doin éd., Paris, 480 p.

ShadiN (W. J.). 1935. - Über die ökologische und geographische Verbreitung der Süsswassermollusken in der U.d. S.S.R. Zoogeographica, 2 : 495-554.

Thibnemann (A.). 1920. - Die Grundlagen der Biocenotik und Monard's faunistische Prinzipien. Festschr. f. Zschohke, 4 : 1-14.

Thienemann (A.). 1950. - Verbreitungsgeschichte der Süsswassertierwelt Europas. Schweizerbart, Stuttgart, 809 p.

Tuffery (G.) in Ixmotte (M.) et Bourlière (F.). 1971. - Problèmes d'Ecologie: l'échantillonnage des peuplements animaux des milieux aquatiques. II. -... L'échantillonnage des peuplements d'Invertébrés des eaux continentales courantes : 53-84. Masson éd., Paris, 294 p. 
Ulfstrand (S.). 1957. - Microdistribution of benthic species (Ephemeroptera, Plecoptera, Trichoptera, Diptera : Simuliidae) in Lapland streams. Oikos, 18 (2) : 293-310.

Verneaux (J.) et Tuffery (G.). 1967. - Une méthode zoologique pratique de détermination de la qualité biologique des eaux courantes. Indices biotiques. Ann. Scient. Univ. Besancon Zool., 3 : 79-90.

Verneaux (J.). 1971 a. - Faune dulçaquicole de Franche-Comté - Le bassin du Doubs (massif du Jura). I : les Poissons; II : les Plécoptères; III : les Trichoptères. Ann. Sci. Univ. Besançon, Zool., $7: 3-46$.

Verneaux (J.). 1971 b. - La larve de Micrasema setiferum Pictet (Trichoptère Brachycentridae) : description et données écologiques Biocénose caractéristique de 17 stations du bassin du Doubs (massif du Jura) et signification typologique. Annls Limnol., 8 (1) : 49-62.

Verneaux (J.). 1972. - Faune dulçaquicole de Franche-Comté - Le bassin du Doubs (massif du Jura). IV : les Ephéméroptères; V : les Odonates. Ann. Sci. Univ. Besançon, Zool., 3 (8) : 3-20.

VERNEAUX (J.). 1973. - Recherches écologiques sur le réseau hydrographique du Doubs - Essai de biotypologie. Thèse Doctorat Sci. Nat., Univ. Besançon. C.N.R.S. A.O. 8823, 260 p., 27 tabl., 61 fig. (Ann. Sci. Univ. Besançon, sous presse).

Wautier (J.). 1949. — Biocœnotique. Soc. Linn. Lyon, 18 (4-5) : 76-95. 